Editorial

\title{
Separations 2019 Best Paper Award
}

\author{
Separations Editorial Office
}

MDPI AG, St. Alban-Anlage 66, 4052 Basel, Switzerland; separations@mdpi.com

Received: 26 February 2020; Accepted: 26 February 2020; Published: 2 March 2020

The Editors of Separations have established the Best Paper Award to acknowledge the authors' support and recognize the most outstanding articles published in the journal.

We are pleased to announce the "Separations Best Paper Awards" for 2019. Papers published in 2017 and 2018 were preselected by the Separations Editorial Office. The awards are issued to reviews and research articles separately. Following a review process by the Editorial Board, the top-voted research article and the top-voted review, which have won the "Separations Best Paper Award" for 2019 are, as follows, in no particular order:

\section{Research Article Award:}

An Alternative Use of Olive Pomace as a Wide-Ranging Bioremediation Strategy to Adsorb and Recover Disperse Orange and Disperse Red Industrial Dyes from Wastewater

Vito Rizzi, Francesco D'Agostino, Jennifer Gubitosa, Paola Fini, Andrea Petrella, Angela Agostiano, Paola Semeraro and Pinalysa Cosma

Separations2017, 4, 29; doi:10.3390/separations4040029

Available online: https://www.mdpi.com/2297-8739/4/4/29

In this paper, the use of olive pomace (OP), a food waste considered highly polluting, for environmental remediation was explored. In particular, hazardous textile industrial dyes chosen as model pollutants, can be sequestered and recovered with excellent performances from wastewater by means of this material. The process was carefully characterized for understanding the nature of interactions involved between dyes and $\mathrm{OP}$, by changing several parameters (contact time, pomace dosage, $\mathrm{pH}$ and temperature values). The principal lignin-cellulosic constituents of OP are deeply involved in the adsorption process through weak and hydrophobic interactions between dyes and the OP pores cavities, with the adsorbed dyes showing a protective role in the thermal degradation of the main OP components. One of the interesting results of this work is the excellent performance of the material at high temperatures-making OP a good biosorbent for real industrial application, since, during the dyeing processes, hot wastewater is generated. Another important aspect is the possibility of recovering dyes by treatment with glacial acetic acid-the auxiliary solvent used during the dyeing processes, enabling the recycling of both of the adsorbent material and dyes with a green and a wide-ranging strategic approach (Figure 1). 


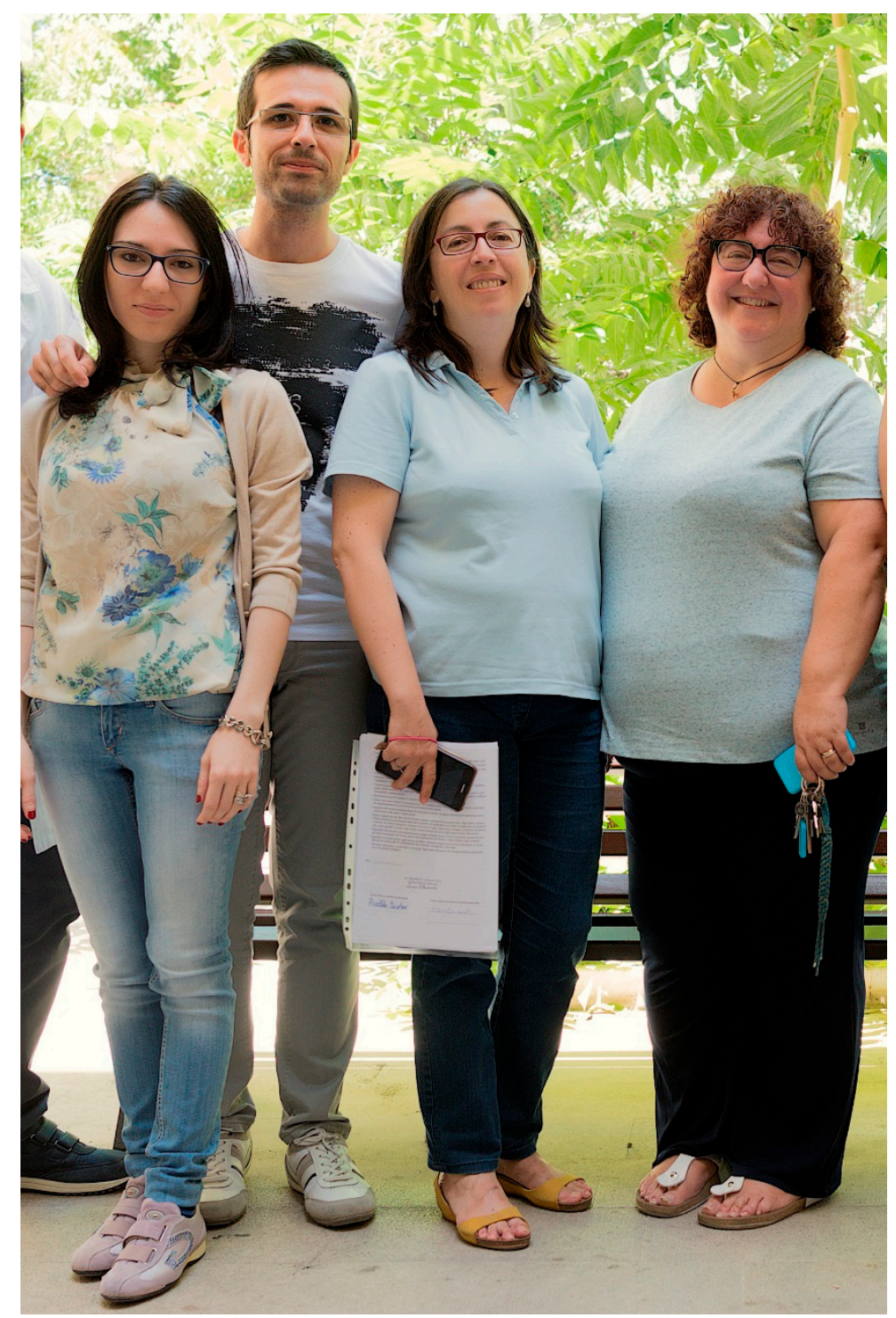

Figure 1. Dr. Pinalysa Cosma's research group.

\section{Review Paper Award:}

Carbon-Based Nanomaterials Functionalized with Ionic Liquids for Microextraction in Sample Preparation

Theodoros Chatzimitakos and Constantine Stalikas

Separations 2017, 4, 14; doi:10.3390/separations4020014

Available online: https://www.mdpi.com/2297-8739/4/2/14

Carbon-based nanomaterials have been extensively used in analytical applications. Fullerenes, carbon nanotubes, nanofibers, nanohorns, graphene and their chemically modified analogues have been investigated as sorbents in sample preparation. Ionic liquids have attracted extensive attention and gained popularity in analytical chemistry, as an alternative to environmentally harmful ordinary organic solvents, covering different application fields like chromatography, electrochemistry and (micro) extraction. The carbon-based nanomaterials modified with ionic liquids possess the advantages of both, and the functionalized materials result in new, advanced adsorbents with tunable microextraction capabilities. 
In this review, attention is paid to the discussion of cases dealing with ionic liquid-functionalized graphene and multi-walled carbon nanotubes, which are examined based on the microextraction mode they support, and we illustrate novel concepts and promising applications (Figure 2).

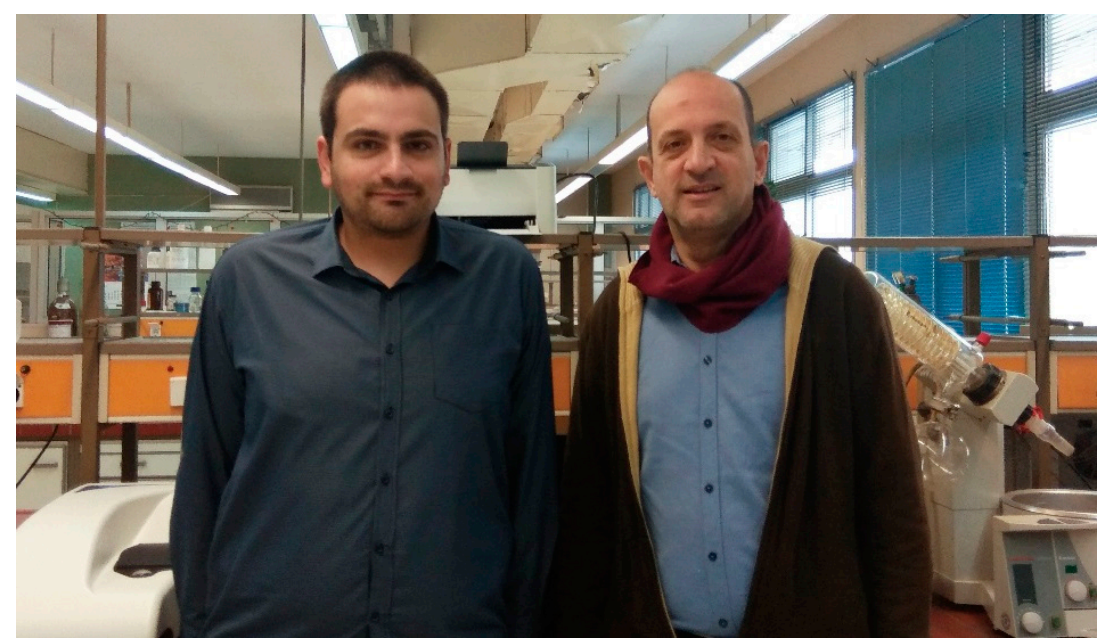

Figure 2. Theodoros Chatzimitakos (left) and Constantine Stalikas (right).

We believe that the two exceptional papers are valuable contributions to Separations and the scientific research field. On behalf of the Separations Editorial Board, we would like to congratulate these teams for their excellent work. In recognition of their accomplishment, they will receive the privilege of publishing an additional research article or review paper free of charge in open access format in Separations, after the usual peer-review procedure.

We would like to take this opportunity to thank all the nominated research groups of the above exceptional papers for their contributions to Separations, and thank the Separations Editorial Board for voting and helping with this "Best Paper Award".

The Editorial Board and Editorial Staff at Separations are committed to meeting your needs by providing useful and timely reviews of all manuscripts submitted, and providing an open access journal for your results. Please consider submitting your work to Separations; we look forward to announcing your paper as a Separations Best Paper in the future.

\section{Prize Awarding Committee}

Separations Editorial Board

(C) 2020 by the author. Licensee MDPI, Basel, Switzerland. This article is an open access article distributed under the terms and conditions of the Creative Commons Attribution (CC BY) license (http://creativecommons.org/licenses/by/4.0/). 